\title{
Plasma Response to Externally Applied Resonant Magnetic Perturbations
}

\author{
Q. Yu and S. Günter
}

Max-Planck-Institut für Plasmaphysik, EURATOM Association, D-85748 Germany

Based on recent finding that applied resonant magnetic perturbation (RMP) can either increase or decrease the local electron density gradient around the resonant surface $(Q . Y u$ and S. Günter 2009 Nucl. Fusion 49 062001), the plasma response to the RMP of a single helicity is studied numerically by further taking into account the electron energy transport. It is found that the changes in the local electron density and temperature by RMPs are coupled. If the local parallel heat diffusivity and/or the island width are sufficiently large, the local electron temperature profile flattens, which enhances the change in the local electron density gradient. Depending on the plasma parameters, either the plasma rotation frequency or the electron diamagnetic frequency (electron pressure gradient) can be significant changed by RMPs, and these two changes affect each other. With a reduced set of equations that does not include the electron energy transport equation, the particle transport in stochastic magnetic fields is found to be similar to that across a single magnetic island. 


\section{Introduction}

Resonant magnetic perturbations (RMPs) are of increasing importance for tokamak plasmas. In addition to intrinsic machine error fields and fields generated by mirror currents in the walls, actively applied RMPs are being used to influence plasma stability. The following issues are of particular concern for a fusion reactor:

(a) Mode locking: The locking of large magnetic islands by error fields or applied RMPs is often observed in tokamak experiments, leading to severe confinement degradation or even to disruptions [1-3]. The neoclassical tearing modes (NTMs) are predicted to be much more easily locked by the error field in a fusion reactor than in existing tokamaks [4,5].

(b) Mitigation of Edge Localized Modes (ELMs): RMPs are found to be able to control ELMs while maintaining the H-mode pedestal [6]. Extensive experimental studies have been carried out to study various parameters affecting the ELMs mitigation, including the plasma shape, beta value, safety factor q profile, and RMP spectrum and amplitude [6-11].

(c) Error field penetration: For the plasma being originally stable to tearing modes, an applied RMP (or error fields of experimental devices) can penetrate through the resonant surface and generate a magnetic island there $[2,12-16]$. The penetrated island could lead to NTMs' onset if its width is sufficiently large. Recent experimental results indicated that the penetration threshold has a minimum when the applied helical field frequency is the same as the mode frequency being determined by both the plasma rotation and the diamagnetic drift [16]. As the field frequency deviates from the mode frequency, the threshold significantly increases and is asymmetric on the two sides of the minimum [16]. Nonlinear numerical results based on two fluids equations agree with the experimental observations [17].

These three issues mentioned above involve the plasma response to external RMPs. There were many theoretical studies in this field based on reduced MHD equations [2,15]. Some phenomena observed in experiments, such as the increased plasma rotation frequency or the change of the plasma rotation from the electron diamagnetic drift direction into the 
ion's direction by an applied static RMP $[2,18]$, can not be explained in this framework. Applied RMPs degrade tokamak particle confinement in many experiments, while improved confinement by RMPs was observed in others [2,19], a phenomenon contradicting the conventional understanding that magnetic islands generated by RMPs would flatten the local plasma density profile [20].

Recently, the plasma response to applied RMPs had been studied by using the reduced nonlinear two-fluid equations [21-29]. It was found that the electromagnetic torque due to the applied RMP drives the plasma rotation towards the ion diamagnetic drift direction with the local rotation frequency approaching the local electron diamagnetic drift frequency [22]. In addition, the local plasma density gradient around the resonant surface can be either increased or decreased by a RMP of a single helicity [23].

In Ref. [23] the electron temperature perturbations caused by RMPs are neglected. In the present paper the electron energy transport is further taken into account, to study the effect of the RMP of a single helicity on the electron density and temperature and plasma rotation velocity. Our first numerical result on the particle transport in stochastic magnetic fields, obtained by neglecting electron temperature perturbations, is also presented.

When the amplitude of the RMP is sufficiently small, the quasi-linear analysis shows that the change of the local plasma density gradient by a static RMP is given by [23]

$$
\mathrm{r}_{\mathrm{s}}\left(\Delta \mathrm{n}_{\mathrm{e}}\right)^{\prime} / \mathrm{n}_{\mathrm{e}}=\left(\chi_{\|} / \mathrm{D}_{\perp}\right)\left(1-\omega_{0}\right)\left|\mathrm{b}_{1 \mathrm{r}} / \mathrm{B}_{0 \mathrm{t}}\right|^{2}\left(\mathrm{r}_{\mathrm{s}} / \mathrm{L}_{\mathrm{pe}}\right)
$$

at the resonant surface $r=r_{s}$ in steady state, where $\Delta n_{e}=\left(n_{e, 0 / 0}-n_{e 0}\right), n_{e, 0 / 0}$ is the $m / n=0 / 0$ component of the electron density $n_{e}$ after applying the RMP, $n_{e}$ is the original equilibrium electron density unperturbed by the RMP, $b_{1 r}$ is the radial magnetic field perturbation, and $\mathrm{B}_{0 \mathrm{t}}$ is the toroidal magnetic field. $\mathrm{D}_{\perp}$ is the perpendicular particle diffusivity, $\chi_{\|}=\mathrm{V}_{\mathrm{Te}}{ }^{2} / \mathrm{v}_{\mathrm{ei}}, \mathrm{V}_{\mathrm{Te}}$ is the electron thermal velocity, $v_{\mathrm{ei}}$ is the electron-ion collisional frequency, and $\mathrm{L}_{\mathrm{pe}}=\mathrm{P}_{\mathrm{e}} / \mathrm{P}_{\mathrm{e}}^{\prime}$ is the scale length of the electron pressure gradient.

$$
\omega_{0} \equiv-\omega_{\mathrm{E} 0} / \omega *_{\mathrm{e} 0}
$$


is the ratio between the equilibrium plasma rotation frequency and the electron diamagnetic drift frequency. $\omega_{0}>0$ refers to the plasma rotation in the ion drift direction (plasma current direction for a toroidal rotation). Equation (1) indicates that a RMP of a single helicity changes the local electron density gradient. The local density gradient decreases $\left(\left|\mathrm{n}_{\mathrm{e}}^{\prime} / \mathrm{n}_{\mathrm{e}}\right|\right.$ increases in the standard case where the equilibrium density gradient is negative) for $\omega_{0}>1$, while in the opposite limit $\left(\Delta \mathrm{n}_{\mathrm{e}}\right)^{\prime}>0$. When the plasma rotation frequency $\omega_{\mathrm{E}}$ and the electron diamagnetic drift frequency $\omega_{*_{\mathrm{e}}}$ are significantly different from $\omega_{\mathrm{E} 0}$ and $\omega_{*_{\mathrm{e}} 0}$, then the $\omega_{\mathrm{E} 0}$ and $\omega *_{\mathrm{e} 0}$ in $\omega_{0}$ should be replaced by $\omega_{\mathrm{E}}$ and $\omega_{*_{\mathrm{e}}}$, respectively [23]. For a plasma with $\mathrm{T}_{\mathrm{e}}=1 \mathrm{keV}$, $\mathrm{n}_{\mathrm{e}}=5 \times 10^{19} \mathrm{~m}^{-3},\left(1-\omega_{0}\right)=-2, \mathrm{~L}_{\mathrm{pe}}=\mathrm{a}, \mathrm{D}_{\perp}=0.1 \mathrm{~m}^{2} / \mathrm{s}$, and $\left|\mathrm{b}_{1 \mathrm{r}} / \mathrm{B}_{0 \mathrm{t}}\right|=10^{-5}$ at $\mathrm{r}=\mathrm{r}_{\mathrm{s}}$, one finds $\left(\Delta \mathrm{n}_{\mathrm{e}}\right)^{\prime} / \mathrm{n}_{\mathrm{e}}=-$ 4.5/a from equation (1), suggesting that the local electron density gradient can be easily changed by a small amplitude of RMP. Nonlinear Numerical results agree with the quasilinear results when the RMP amplitude is not too large [23].

With further taking into account the electron energy transport, it is found in this paper that the electron temperature changes in a similar way to the electron density, if the local parallel heat diffusivity and the island width are not too large. In the opposite case the local electron temperature profile flattens, which enhances the change in the local electron density gradient. It is also found that depending on the plasma parameters, either the plasma rotation frequency or the electron diamagnetic frequency (electron pressure gradient) can be significant changed by RMPs, and these two changes affect each other. The particle transport in stochastic magnetic fields, obtained by neglecting electron temperature perturbations, is found to be similar to that across a single magnetic island.

\section{Theoretical model}

The large aspect-ratio tokamak approximation is utilized. The magnetic field is defined as $B=B_{0 t} \mathbf{e}_{t^{-}}(\mathrm{kr} / \mathrm{m}) \mathrm{B}_{0 \mathrm{t}} \mathbf{e}_{\theta}+\nabla \psi \times \mathbf{e}_{\mathrm{t}}$, where $\psi$ is the helical flux function, $\mathrm{m} / \mathrm{r}$ and $\mathrm{k}=\mathrm{n} / \mathrm{R}$ are the 
wave vectors in $\mathbf{e}_{\theta}$ (poloidal) and $\mathbf{e}_{\mathrm{t}}$ (toroidal) direction, respectively, $\mathrm{R}$ is the major radius, and the subscript 0 denotes an equilibrium quantity. The ion velocity $\mathrm{v}^{=\mathrm{v}_{\|}} \mathbf{e}_{\|}+\mathbf{v}_{\perp}$, where $\mathbf{v}_{\|}$and $\mathbf{v}_{\perp}=\nabla \phi \times \mathbf{e}_{\mathrm{t}}$ are the parallel (to the magnetic field) and the perpendicular velocity, respectively. The cold ion assumption is made as in Ref. [23].

To obtain $\psi, \mathbf{v}_{\|}, \mathbf{v}_{\perp}$, the electron density $\mathrm{n}_{\mathrm{e}}$ and temperature $\mathrm{T}_{\mathrm{e}}$, the electron continuity equation, the generalized Ohm's law, the equation of motion in the parallel and the perpendicular direction (after taking the operator $\mathbf{e}_{t} \nabla \times$ ), and the electron energy transport equation, are solved [30]. Normalizing the length to the minor radius a, the time $t$ to $\tau_{\mathrm{R}}, \psi$ to $\mathrm{aB}_{0 t}, \mathbf{v}$ to $\mathrm{a} / \tau_{\mathrm{R}}$, and $\mathrm{T}_{\mathrm{e}}$ and $\mathrm{n}_{\mathrm{e}}$ to their values at the magnetic axis, where $\mathrm{a}$ is the minor radius, and $\tau_{\mathrm{R}}=\mathrm{a}^{2} / \eta$ is the resistive time, these equations become [30]

$$
\begin{aligned}
& \frac{d n_{e}}{d t}=d_{1} \nabla_{\|} j-\nabla_{\|}\left(n_{e} v_{\|}\right)+\nabla \cdot\left(D_{\perp} \nabla n_{e}\right)+S_{n}, \\
& \frac{d \psi}{d t}=E-\eta j+\Omega\left(\nabla_{\|} n_{e}+\nabla_{\|} T_{e}\right), \\
& \frac{d v_{\|}}{d t}=-C_{s}^{2} \nabla_{\|} P / n_{e}+\mu \nabla_{\perp}^{2} v_{\|}, \\
& \frac{d U}{d t}=-S^{2} \nabla_{\|} j+\mu \nabla_{\perp}^{2} U+S_{m}, \\
& \frac{3}{2} n_{e} \frac{d T_{e}}{d t}=d_{1} T_{e} \nabla_{\|} j-T_{e} n_{e} \nabla_{\|} v_{\|}+n_{e} \nabla \cdot\left(\chi_{\|} \nabla_{\|} T_{e}\right)+n_{e} \nabla \cdot\left(\chi_{\perp} \nabla_{\perp} T_{e}\right)+S_{p},
\end{aligned}
$$

where $\mathrm{d} / \mathrm{dt}=\partial / \partial \mathrm{t}+\mathbf{v}_{\perp} \cdot \nabla, \mathrm{j}=-\nabla_{\perp}^{2} \psi-2 \mathrm{kB}_{0 \mathrm{t}} / \mathrm{m}$ is the plasma current density along the $\mathbf{e}_{\mathrm{t}}$ direction, $\mathrm{U}=-\nabla_{\perp}^{2} \phi$ is the plasma vorticity, $\mu$ the plasma viscosity, $\chi$ the heat conductivity, and $\mathrm{D}$ the particle diffusivity. $\mathrm{P}=\mathrm{P}_{\mathrm{e}}=\mathrm{n}_{\mathrm{e}} \mathrm{T}_{\mathrm{e}}$, and the subscripts $\|$ and $\perp$ denote the parallel and the perpendicular components, respectively. $S_{n}$ and $S_{p}$ are the particle and heat source. $E=\eta j_{0}$ is the equilibrium electric field for maintaining the original equilibrium plasma current density $\mathrm{j}_{0} . \mathrm{S}_{\mathrm{m}}=-\mu \nabla_{\perp}^{2} \mathrm{U}_{0}=\mathrm{S}_{\mathrm{m} 0}\left[1-(\mathrm{r} / \mathrm{a})^{2}\right]^{3}$ is the poloidal momentum source which leads to an equilibrium 
poloidal plasma rotation, $\mathrm{U}_{0}$ is the equilibrium plasma vorticity without $\mathrm{RMP}$, and $\mathrm{S}_{\mathrm{m} 0}$ is a constant to define the source amplitude. The parameters in Equation (2)-(6) are given by $\mathrm{d}_{1}=\omega_{\text {ce }} / v_{\mathrm{ei}}, \Omega=\beta_{\mathrm{e}} \mathrm{d}_{1}, \mathrm{C}_{\mathrm{s}}=\left[\mathrm{T}_{\mathrm{e}} / \mathrm{m}_{\mathrm{i}}\right]^{1 / 2} /\left(\mathrm{a} / \tau_{\mathrm{R}}\right)$, and $\mathrm{S}=\tau_{\mathrm{R}} / \tau_{\mathrm{A}}$, where $\beta_{\mathrm{e}}=4 \pi \mathrm{n}_{\mathrm{e}} \mathrm{T}_{\mathrm{e}} / \mathrm{B}_{0 \mathrm{t}}{ }^{2}, \omega_{\text {ce }}$ is the electron cyclotron frequency, and $\tau_{\mathrm{A}}=\mathrm{a} / \mathrm{V}_{\mathrm{A}}$ is the toroidal Alfven time.

Equations (2)-(5) are the same as those utilized in Reference [23], based on the fourfield model equations [31]. Equation (6) further takes into account the change in the electron temperature by RMPs, which is a simplified form from two-fluid equations [32,33]. Both the parallel and the perpendicular heat transport are known to be important in determining the electron temperature profile across a magnetic island [34]. Only a constant plasma viscosity is taken for simplicity, assuming to be caused by anomalous momentum transport due to plasma turbulence.

Equations (2)-(6) are solved simultaneously using the initial value code TM1, which has been used earlier for modelling drift tearing modes [30]. Dedicated numerical methods are utilized in the code to reduce the numerical error caused by large values of $\chi_{\|}$[35]. The effect of the RMP is taken into account by the boundary condition

$$
\psi_{\mathrm{m} / \mathrm{n} \mid \mathrm{r}=\mathrm{a}}=\psi_{\mathrm{aaB}} \mathrm{B}_{0+\mathrm{c}} \cos (\mathrm{m} \theta+\mathrm{n} \phi)
$$

where $\psi_{\mathrm{a}}$ describes the normalized helical magnetic flux amplitude of the $\mathrm{m} / \mathrm{n}$ component at $\mathrm{r}=\mathrm{a}$. The radial magnetic field perturbation at $\mathrm{r}=\mathrm{a}$ is given by $\mathrm{b}_{\mathrm{lr}}=-\mathrm{m} \psi_{\mathrm{a}} \mathrm{B}_{0 \mathrm{t}} \sin (\mathrm{m} \theta+\mathrm{n} \phi)$.

\section{Numerical results}

The electron temperature and density are quite different for different tokamak plasmas and/or with different additional heating power or fueling rate. Even in a single tokamak discharge, they can change by an order of magnitude from the edge to the core region. The perpendicular transport coefficients in the pedestal region of H-mode plasmas are also quite different from those in the core region. Therefore, the values of these parameters in equations (2)-6), such as $S, d_{1}$, and the transport coefficients, can vary by orders of magnitude. In order 
to have a more general understanding and to identify the key physics and parameters affecting the plasma response to RMPs, our calculations have been carried out over a large range of values for these parameters. In the following Section 3.1-3.3 we focus on the change of the electron temperature and density by RMPs, and in this case the change in the plasma rotation frequency is not significant. The change of the plasma rotation by RMPs is further studied in Section 3.4 by using different plasma viscosity. The particle transport in stochastic magnetic fields is investigated in Section 3.5 with a reduced set of equations that does not include the electron energy transport equation.

\subsection{Effect of RMP amplitude and parallel heat diffusion}

The following parameters, the plasma minor $\mathrm{a}=0.47 \mathrm{~m}$, the major radius $\mathrm{R}=1.75 \mathrm{~m}$, $\mathrm{S}=1.97 \times 10^{8}, \Omega=6.3 \times 10^{4}, \quad \mathrm{c}_{\mathrm{s}}=1 \times 10^{7}\left(\mathrm{a} / \tau_{\mathrm{R}}\right), \quad \mathrm{d}_{1}=2.5 \times 10^{8}, \quad \chi_{\|}=10^{8}\left(\mathrm{a}^{2} / \tau_{\mathrm{R}}\right), \quad \chi_{\perp}=\mathrm{D}_{\perp}=21\left(\mathrm{a}^{2} / \tau_{\mathrm{R}}\right), \quad$ and $\mu=2.1 \times 10^{3}\left(\mathrm{a}^{2} / \tau_{\mathrm{R}}\right)$, are used except mentioned elsewhere. In tokamak experiments the plasma rotation is usually toroidal [2,15], while in Equation (2)-(6) due to large aspect ratio approximation only the poloidal rotation is included, so that a larger plasma viscosity is used here for a reasonable balance between the electromagnetic and viscous force $[2,23]$. The change in the local electron density gradient is larger for a larger value of $d_{1} / D_{\perp N}$, where $\mathrm{D}_{\perp \mathrm{N}}=\mathrm{D}_{\perp} /\left(\mathrm{a}^{2} / \tau_{\mathrm{R}}\right)$ [23]. Above parameters lead to $\mathrm{d}_{1} / \mathrm{D}_{\perp \mathrm{N}}=1.19 \times 10^{7}$, corresponding to a electron density $\mathrm{n}_{\mathrm{e}}=10^{19} \mathrm{~m}^{-3}$ for $\mathrm{D}_{\perp}=0.33 \mathrm{~m}^{2} / \mathrm{s}$ and $\mathrm{B}_{0 \mathrm{t}}=2.25 \mathrm{~T}$ or to a higher electron density for a stronger toroidal field, as $d_{1} / D_{\perp N}$ is proportional to $B_{0 t} /\left(n_{e} D_{\perp}\right)$. Only a single helicity RMP with $\mathrm{m} / \mathrm{n}=2 / 1$ is taken into account here. A monotonic q-profile is used with the $\mathrm{q}=2$ surface located at $\mathrm{r}_{\mathrm{s}}=0.628 \mathrm{a}$. The $\mathrm{m} / \mathrm{n}=2 / 1$ tearing mode is stable for $\psi_{\mathrm{a}}=0$.

The radial profiles of the (normalized) $\mathrm{m} / \mathrm{n}=0 / 0$ component of the electron temperature, $\mathrm{T}_{\mathrm{e}, 0 / 0} / \mathrm{T}_{0}$, in steady state are shown by the solid curves in Fig. 1 a for $\omega_{0}=2.97$ with $\psi_{\mathrm{a}}=8 \times 10^{-5}$, $2 \times 10^{-4}$ and $3 \times 10^{-4}$, where $\mathrm{T}_{0}$ is the equilibrium electron temperature at $\mathrm{r}=0$. The corresponding 
magnetic island widths are w/a $=0.0847,0.116$ and 0.126 , respectively. The dotted curve shows the radial profile of the original equilibrium electron temperature. The electron temperature increases across the rational surface for $\psi_{\mathrm{a}}=8 \times 10^{-5}$, forming a kind of pedestal there as expected, since the $\nabla_{\mid \mathrm{j}}$ term in equation (6) can lead to a similar increase in the local electron temperature as in the electron density for $\omega_{0}>1$ [23]. For larger $\psi_{\mathrm{a}}$, the electron temperature decreases, suggesting the possible role of the parallel heat diffusion for a sufficiently large magnetic island [34]. Corresponding to Fig. 1a, radial profiles of the (normalized) $\mathrm{m} / \mathrm{n}=0 / 0$ component of the electron density, $\mathrm{n}_{\mathrm{e}, 0 / 0} / \mathrm{n}_{0}$, in steady state are shown by the solid curves in Fig. $1 b$, where $n_{0}$ is the equilibrium electron density at $r=0$. The dotted curve shows the equilibrium electron density profile. The local electron density increases more significantly for a larger value of $\psi_{\mathrm{a}}$. This could be explained as the following: The decreases in the electron temperature gradient results in a smaller local electron diamagnetic drift frequency and therefore a larger difference between this frequency and the local plasma rotation frequency, leading to a corresponding larger effect of the $\nabla_{\| j}$ term in equation (2) or a larger value of $\left|1-\omega_{0}\right|$ in equation (1), so that the electron density gradient changes more significantly accompanying the decreases in the electron temperature.

For the plasma rotation in the electron drift direction with $\omega_{0}=-1.98$, radial profiles of $\mathrm{T}_{\mathrm{e}, 0 / 0} / \mathrm{T}_{0}$ in steady state are shown in figure $2 \mathrm{a}$ with $\psi_{\mathrm{a}}=8 \times 10^{-5}, 10^{-4}$ and $3 \times 10^{-4}$. The corresponding island widths are $\mathrm{w} / \mathrm{a}=0.0125,0.0884$ and 0.130 , respectively. The dotted curve shows again the equilibrium electron temperature profile. With increasing $\psi_{\mathrm{a}}$, the electron temperature first decreases, and the local temperature gradient changes from the usual negative value to a positive one. For sufficiently large $\psi_{\mathrm{a}}$, the temperature profile flattens. Corresponding to Fig. 2a, radial profiles of $\mathrm{n}_{\mathrm{e}, 0 / 0} / \mathrm{n}_{0}$ are shown in Fig. $2 b$. The electron density decreases more significantly across the rational surface for a larger value of $\psi_{\mathrm{a}}$ accompanying the flattening in the electron temperature profile. 
In order to look into the effect of the parallel electron heat diffusion, corresponding to figure 2 with $\omega_{0}=-1.98$, the electron temperature profiles are shown in figure $3 \mathrm{a}$ for $\psi_{\mathrm{a}}=10^{-4}$, with $\chi_{\|}=10^{7}, 10^{8}$ and $1.6 \times 10^{9}$. The corresponding island widths are $\mathrm{w} / \mathrm{a}=0.0886,0.0884$, and 0.0859, respectively. With increasing $\chi_{\| 1}$, the local temperature profile becomes more flattened. The corresponding electron density (Fig. 3b) decreases more significantly across the rational surface for a larger $\chi_{\|}$with the flattening of the local temperature profile, being similar to those shown in figure $2 \mathrm{~b}$ for a larger $\psi_{\mathrm{a}}$.

Corresponding to figure 1 with $\omega_{0}=2.97$, the electron temperature profiles in steady state are shown in figure $4 \mathrm{a}$ for $\psi_{\mathrm{a}}=8 \times 10^{-5}$ with $\chi_{\|}=10^{7}, 10^{8}$ and $10^{9}$. The magnetic island widths are $\mathrm{w} / \mathrm{a}=0.0877,0.0847$ and 0.0823 , respectively. The local temperature increases for $\chi_{\|}$up to $10^{8}$ but decreases for $\chi_{\|}=10^{9}$. The corresponding electron density (Fig. 4b) increases more significantly across the rational surface for a lager $\chi_{\|}$.

It is seen from Figs. 1-4 that the change of the local gradient of the electron temperature and density by RMPs is coupled. The flattening in the local electron temperature profile due to a large value of $\chi_{\|}$or magnetic island width enhances the change of the electron density gradient.

\subsection{Effect of original equilibrium plasma rotation direction and frequency}

Figures 1-4 have already shown the significant difference in the plasma response to RMPs with different equilibrium plasma rotation direction. A more detailed result about the effect of original equilibrium plasma rotation direction and frequency is shown in figures $5 \mathrm{a}$ and $5 b$.

The radial profiles of the electron temperature, $\mathrm{T}_{\mathrm{e}, 0 / 0} / \mathrm{T}_{0}$, in steady state are shown by the solid curves in Fig. 5a for $\psi_{\mathrm{a}}=8 \times 10^{-5}$ with $\omega_{0}=2.97,1.0,-1.98$ and 0 . The island widths are $\mathrm{w} / \mathrm{a}=0.0847,0.0778,0.0125$ and 0.0756 , respectively. The corresponding electron density 
profiles are shown in Fig. 5b. The electron temperature and density increase across the rational surface for $\omega_{0}>1$ but decreases for $\omega_{0}<1$ as expected, since the parallel heat diffusivity $\left(\chi_{\|}=10^{8}\right)$ is not too large. For $\omega_{0}=1.0$, the electron temperature decreases across the rational surface due to the parallel heat diffusion, and the $\nabla_{\mid \mathrm{j}}$ term in equation (6) can be neglected in this case. The change in the electron temperature gradient results in a smaller local electron diamagnetic drift frequency, leading to $-\omega_{\mathrm{E}} / \omega_{*}>1\left(\omega_{0}>1\right)$ and therefore a slight increase in the electron density.

It is seen from figures 1-5 that the parallel heat diffusion causes the different change in the local electron temperature and density gradient by RMPs. A decrease in the electron temperature corresponds to an increase in the electron density.

\subsection{Effect of $d_{1}$ and perpendicular particle diffusivity}

The effect of the parameter $d_{1}$ on the electron temperature and density profiles is shown in figures $6 \mathrm{a}$ and $6 \mathrm{~b}$ for $\omega_{0}=2.97$ and $\psi_{\mathrm{a}}=8 \times 10^{-5}$, with $\mathrm{d}_{1}=2.5 \times 10^{8}, 1.5 \times 10^{8}$, and $8 \times 10^{7}$. The island widths are $\mathrm{w} / \mathrm{a}=0.0847,0.0830$ and 0.0758 , respectively. As $\omega_{0}>1$ in this case, the electron temperature and density increase more across the rational surface for a larger value of $d_{1}$ as expected from equation (1). For the plasma rotation in the electron drift direction, a larger value of $d_{1}$ is also found to lead to a larger change in the local electron temperature and density gradient when the parallel heat diffusivity and the island width are not too large.

The effect of the perpendicular particle and heat diffusivity on the electron temperature and density profiles is shown in figures $7 \mathrm{a}$ and $7 \mathrm{~b}$ for $\omega_{0}=2.97$ and $\psi_{\mathrm{a}}=8 \times 10^{-5}$, with $\chi_{\perp}=\mathrm{D}_{\perp}=21$ and $63\left(\mathrm{a}^{2} / \tau_{\mathrm{R}}\right)$. The island widths are $\mathrm{w} / \mathrm{a}=0.0847$ and 0.0755 , respectively. The electron density increases more across the rational surface for a smaller value of $\mathrm{D}_{\perp}$ as expected [23]. The electron temperature, however, increases more for a larger value of $D_{\perp}\left(\chi_{\perp}\right)$, being similar to that shown figure $4 \mathrm{a}$ for a smaller $\chi_{\|}$, since the parallel heat diffusion is not efficient for a 
smaller value of $\chi_{\|} / \chi_{\perp}$. For $\mathrm{D}_{\perp}=63\left(\mathrm{a}^{2} / \tau_{\mathrm{R}}\right)$ one has $\mathrm{d}_{1} / \mathrm{D}_{\perp \mathrm{N}}=3.97 \times 10^{6}$, corresponding to an electron density $n_{e}=10^{19} \mathrm{~m}^{-3}$ for $\mathrm{D}_{\perp}=1.0 \mathrm{~m}^{2} / \mathrm{s}$ and $\mathrm{B}_{0 \mathrm{t}}=2.25 \mathrm{~T}$.

\subsection{Change of plasma rotation frequency by RMP}

In addition to the change in the local electron density and temperature gradient, the electromagnetic torque due to the applied RMP drives the plasma rotation frequency $\omega_{\mathrm{E}}$ to approach the negative electron diamagnetic drift frequency $\left(\omega_{\mathrm{E}} \approx-\omega_{*_{\mathrm{e}}}\right)$ [22]. For the results presented in Section 3.1-3.3, the change of the plasma rotation frequency is not very significant. The effect of RMPs on plasma rotation is determined by the balance between the electromagnetic and viscous torque, which is affected by the parameter S and the normalized plasma viscosity $\left(\mu \tau_{\mathrm{R}} / \mathrm{a}^{2}\right)$ as seen from Eq. (5). A larger value of $S^{2} /\left(\mu \tau_{\mathrm{R}} / \mathrm{a}^{2}\right)$ leads to a bigger change in plasma rotation. In the following results the value of $S$ is fixed, and only the normalized plasma viscosity is changed in order to vary the value of $S^{2} /\left(\mu \tau_{R} / a^{2}\right)$. Other parameters are kept unchanged.

The (normalized) $\mathrm{m} / \mathrm{n}=0 / 0$ component of the poloidal plasma rotation velocity, $V_{\mathrm{p}}$, is shown in figure $8 \mathrm{a}$ for $\omega_{0}=2.97$ and $\psi_{\mathrm{a}}=8 \times 10^{-5}$, with $\mu=2100,210$ and $21\left(\mathrm{a}^{2} / \tau_{\mathrm{R}}\right)$. The corresponding island widths are $\mathrm{w} / \mathrm{a}=0.0847,0.0871$ and 0.0740 , respectively. The dotted curve shows the original poloidal velocity profile unperturbed by the RMP. With decreasing $\mu$, the plasma rotation velocity decreases more. Corresponding to Fig. 8a, radial profiles of $\mathrm{T}_{\mathrm{e}, 0 / 0} / \mathrm{T}_{0}$ and $\mathrm{n}_{\mathrm{e}, 0 / 0} / \mathrm{n}_{0}$ are shown in Fig. $8 \mathrm{~b}$ and $8 \mathrm{c}$. The local electron temperature and density are increased for a large value of $\mu$ but decreased across the rational surface for a sufficiently small $\mu$. It is seen that the change of the plasma rotation frequency affects the change in the local electron temperature and density gradient.

For the plasma rotation in the electron drift direction with $\omega_{0}=-1.98$, radial profiles of $\mathrm{V}_{\mathrm{p}}$ are shown in figure $9 \mathrm{a}$ with $\psi_{\mathrm{a}}=10^{-4}$ for $\mu=2100,210$ and $21\left(\mathrm{a}^{2} / \tau_{\mathrm{R}}\right)$. The corresponding island 
widths are $\mathrm{w} / \mathrm{a}=0.0857,0.0871$ and 0.0740 , respectively. With decreasing $\mu$, the plasma rotation velocity first decreases and then changes its direction from the electron drift direction to the ion's direction around the rational surface. The corresponding radial profiles of $\mathrm{T}_{\mathrm{e}, 0 / 0} / \mathrm{T}_{0}$ and $\mathrm{n}_{\mathrm{e}, 0 / 0} / \mathrm{n}_{0}$ are shown in Fig. $9 \mathrm{~b}$ and $9 \mathrm{c}$. The electron temperature and density decrease less across the rational surface for a smaller $\mu$.

For zero equilibrium plasma rotation, the radial profiles of $V_{p}$ in steady state are shown in figure $10 \mathrm{a}$ with $\psi_{\mathrm{a}}=8 \times 10^{-5}$ for $\mu=2100,210$ and $21\left(\mathrm{a}^{2} / \tau_{\mathrm{R}}\right)$. The corresponding island widths are $\mathrm{w} / \mathrm{a}=0.0756,0.0765$ and 0.0748 . The RMP drives the plasma to rotate in the ion drift direction, in agreement with the experimental observations [18]. With decreasing $\mu$, the plasma rotation velocity increases more. Corresponding to Fig. $10 \mathrm{a}$, radial profiles of $\mathrm{T}_{\mathrm{e}, 0 / 0} / \mathrm{T}_{0}$ and $\mathrm{n}_{\mathrm{e}, 0 / 0} / \mathrm{n}_{0}$ are shown in Fig. 10b and 10c. The electron temperature and density decrease less across the rational surface for a smaller $\mu$.

It is seen from Figs. 8-10 that the plasma response to the RMP is affected by the values of $\mathrm{S}^{2} /\left(\mu \tau_{\mathrm{R}} / \mathrm{a}^{2}\right)$. When this value is sufficiently large, RMPs lead to a more significant change in the plasma rotation frequency than the local electron diamagnetic drift frequency (electron pressure gradient). A larger change in the plasma rotation velocity corresponds to a smaller change in the local electron pressure gradient.

\subsection{Particle transport across a local stochastic magnetic field}

The local magnetic field becomes stochastic when islands of different helicity overlap. An applied $\mathrm{m} / \mathrm{n}=9 / 5 \mathrm{RMP}$ is further introduced in our calculation in addition to the $\mathrm{m} / \mathrm{n}=2 / 1$ RMP, with the $\mathrm{q}=9 / 5$ surface at $\mathrm{r}_{\mathrm{s}}=0.593 \mathrm{a}$, being close to the $\mathrm{q}=2$ surface at $\mathrm{r}_{\mathrm{s}}=0.628 \mathrm{a}$.

In the following results the electron temperature perturbations are neglected, and only the particle transport is calculated by using Equations (2)-(5) due to computational limitation. In Fig. 11a radial profiles of $\mathrm{n}_{\mathrm{e}, 0 / 0} / \mathrm{n}_{0}$ in steady state are shown for $\omega_{0}=2.92$ with (1) $\psi_{\mathrm{a}, 2 / 1}=$ 
$1.8 \times 10^{-5}$ and $\psi_{\mathrm{a}, 9 / 5}=8 \times 10^{-4} ;(2) \psi_{\mathrm{a}, 2 / 1}=10^{-4}$ and $\psi_{\mathrm{a}, 9 / 5}=10^{-3}$. The Chirikov parameters are $\Delta=0.94$ and 1.55 , respectively, calculated only by the $m / n=/ 2 / 1$ and $9 / 5$ islands. For Curve (2) the island width are $\mathrm{w}_{2 / 1}=0.0847 \mathrm{a}$ and $\mathrm{w}_{9 / 5}=0.0239 \mathrm{a}$. In fact, many small islands of other helicities are also excited by the applied $\mathrm{m} / \mathrm{n}=2 / 1$ and 9/5 RMPs, e.g., $\mathrm{w}_{11 / 6}=0.00413 \mathrm{a}, \mathrm{w}_{13 / 7}=0.0013 \mathrm{a}$, $\mathrm{w}_{15 / 8}=0.0024 \mathrm{a}, \mathrm{w}_{17 / 9}=0.0021 \mathrm{a}, \mathrm{w}_{7 / 4}=0.0070 \mathrm{a}, \mathrm{w}_{5 / 3}=0.0042 \mathrm{a}$, and etc. Fig. $11 \mathrm{~b}$ is for the original equilibrium plasma rotation in the electron drift direction with $\omega_{0}=-1.62$ for (1) $\psi_{\mathrm{a}, 2 / 1}=2 \times 10^{-5}$ and $\psi_{\mathrm{a}, 9 / 5}=9 \times 10^{-4} ;$ (2) $\psi_{\mathrm{a}, 2 / 1}=10^{-4}$ and $\psi_{\mathrm{a}, 9 / 5}=9 \times 10^{-4}$. The Chirikov parameters are $\Delta=0.981$, and 1.53 respectively. The electron density increases across the rational surface for the plasma rotation in the ion drift direction but decreases in the opposite case, being similar to that caused by a single helicity RMP [23].

In above calculations in stochastic field the parallel resistivity is defined according to Spitzer expression, being valid for high collisionality. It was shown that this expression is modified for low collisionality plasmas [29].

\section{Discussion and summary}

Above results indicate that the plasma response to RMPs can be quite different for different plasma parameters. Depending on the value of $\mathrm{S}^{2} /\left(\mu \tau_{\mathrm{R}} / \mathrm{a}^{2}\right)$, either the plasma rotation frequency or the electron diamagnetic drift frequency (electron temperature and density gradient) can be significantly changed by RMPs. As $S^{2} /\left(\mu \tau_{R} / a^{2}\right) \sim T_{e}^{3 / 2} B_{0 t}{ }^{2} /\left(\mu n_{e}\right)$, being larger in the tokamak core region where the electron temperature is higher, one would expected a significant change in the plasma rotation frequency there by RMPs. While in the edge region where the local electron temperature is low, the local electron density and temperature gradient can also be significantly changed.

It should be mentioned that our results are obtained with fixed perpendicular particle and heat diffusivity. The increase of local rotation velocity shear by RMPs due to the electromagnetic torque can possibly decrease the local plasma turbulence level and 
perpendicular particle diffusivity [36]. The neoclassical torque has not been included in equation (5), which could be important in the pedestal region of H-mode plasmas [24]. It has been shown that the neoclassical toroidal viscosity (NTV) due to non-axisymmetric field drives the plasma to rotate in the electron diamagnetic direction with a frequency approaching the ion diamagnetic drift frequency [37]. In the presence of a sufficiently strong NTV effect such that the plasma is forced to rotate in the electron diamagnetic direction, our results suggest that the RMP will decreases the plasma density, especially for the plasma with a higher parallel heat diffusivity as seen from figure 3 .

In summary, the plasma response to RMPs has been shown to be affected by the plasma rotation direction and frequency, the RMP amplitude, and the particle, heat and momentum transport coefficients. The major obtained results are as the following:

(1) In case of a sufficiently high value of $S^{2} /\left(\mu \tau_{R} / a^{2}\right)$, the change of the plasma rotation frequency is more significant than that of the local electron density and temperature gradient (electron diamagnetic drift frequency). The RMP can either speed up or slow down the plasma rotation or even change the rotation direction, depending on the original equilibrium plasma rotation frequency and direction.

(2) In case of a sufficiently low value of $S^{2} /\left(\mu \tau_{R} / a^{2}\right)$, the change of the local electron density gradient can be significant. The RMP can either increase or decrease the local electron density gradient, depending on the original equilibrium plasma rotation frequency and direction.

(3) The electron temperature changes in a similar way to the electron density, if the local parallel heat diffusivity and the island width are not too large. In the opposite case the local electron temperature profile flattens, which enhances the change in the local electron density gradient.

(4) The particle transport in stochastic magnetic fields is found to be similar to that caused by a single helicity RMP. 
Acknowledgement: Q. Yu is grateful to Dr. K. H. Finken for helpful discussions. 


\section{Reference}

[1] M.F.F. Nave and J.A. Wesson 1990 Nucl. Fusion 302575

[2] T.C. Hender, R. Fitzpatrick, A.W. Morris et al 1992 Nucl. Fusion 322091

[3] H. Zohm, A. Kallenbach, H. Bruhns, et al 1990 Europhys. Letts. 11745

[4] Q. Yu and S. Günter, 2008 Nucl. Fusion 48065004

[5] La Haye R.J. et al 2006 Nucl. Fusion 46451

[6] T.E. Evans, R.A. Moyer, P.R. Thomas, et al 2004 Phys. Rev. Letts. 92235003

[7] Y. Liang, et al 2007 Phys. Rev. Lett. 98265004

[8] T.E. Evans et al 2008 Nucl. Fusion 48024002

[9] T.E. Evans et al 2006 Phys. Plasmas 13, 056121

[10] M.E. Fenstermacher et al 2008 Phys. Plasmas 15, 056122

[11] M.E. Fenstermacher et al 2008 Nucl. Fusion 48122001

[12] R. J. La Haye, R. Fitzpatrick, T. C. Hender, et al 1992 Phys. Fluids B 42098

[13] R.J. Buttery, M. De' Benedetti, T.C. Hender and B.J.D. Tubbing, 2000 Nucl. Fusion 40 807

[14] S. M. Wolfe, I. H. Hutchinson, R. S. Granetz, J. Rice, and A. Hubbard, 2005 Phys. Plasmas 12056110

[15] R. Fitzpatrick, 1993 Nucl. Fusion 331049

[16] H.R. Koslowski, Y. Liang A. Krämer-Flecken et al 2006 Nucl. Fusion 46 L1

[17] Q. Yu, S. Günter, Y. Kikuchi, and K. H. Finken, 2008 Nuclear Fusion 48024007

[18] K.H. Finken, S.S.Abdullaev, M.F.M. De Bock et al 2005 Phys. Rev. Letts 94015003

[19] K.H.Finken, S.S.Abdullaev, M.W.Jakubowski et al 2007 Phys. Rev. Letts 98065001

[20] Liewer P.C. 1985 Nucl. Fusion 25543

[21] F. L. Waelbroeck, 2003 Phys. Plasmas 104040

[22] Q. Yu, S. Günter and K.H. Finken, 2009 Phys. Plasmas 16042301

[23] Q. Yu and S. Günter, 2009 Nucl. Fusion 49062001 
[24] M. Becoulet et al Proc. 37th EPS Conf. on Contr. Fus. \& Plasma Phys. Dublin Vol. 34A, ISBN 2-914771-62-2 (2010) P-4.105. http://ocs.ciemat.es/EPS2010PAP/pdf/P4.105.pdf

[25] M. Becoulet, G. Huysmans, X. Garbet et al 2009 Nucl. Fusion 49085011

[26] E. Nardon,P. Tamain, M. Becoulet et al 2010 Nucl. Fusion 50034002

[27] V. Rozhansky et al 2010 Nucl. Fusion 50034005

[28] M. Z. Tokar et al 2008 Phys. Plasmas 15072515.

[29] I. Kaganovich and V. Rozhansky 1998 Phys. Plasmas 53901

[30] Q. Yu, 2010 Nucl. Fusion 50025014

[31] R.D.Hazeltine, M. Kotschenreuther and P.J. Morrison, 1985 Phys Fluids 282466

[32] Braginskii S. I. Reviews of Plasma Physics, 1965 Vol. 1, 205, edited by M Leontovich (New York Consultants Bureau)

[33] Smolyakov A.I. 1993 Plasma Phys. Control. Fusion 35657

[34] Q. Yu, 2006 Phys. Plasmas 13062310

[35] S. Günter, Q. Yu, J. Krüger, and K. Lackner, 2005 J. Comp. Phys. 209354

[36] O. Schmitz, J.W. Coenen, H. Frerichs, et al 2009 J. Nucl. Materials 390-391 330

[37] J.D. Callen, A.J. Cole and C.C. Hegna, 2009 Nucl. Fusion 49085021 


\section{Caption}

Fig. 1: Effect of the RMP amplitude on radial profiles of the (normalized) $\mathrm{m} / \mathrm{n}=0 / 0$ component of electron temperature (1a) and density (1b). The dotted curves show the radial profiles of the original equilibrium electron temperature and density. The equilibrium plasma rotation is in the ion drift direction with $\omega_{0}=2.97$.

Fig. 2: Effect of the RMP amplitude on radial profiles of the (normalized) $m / n=0 / 0$ component of electron temperature (2a) and density (2b) . The original equilibrium plasma rotation is in the electron drift direction with $\omega_{0}=-1.98$.

Fig. 3: Effect of parallel heat diffusion on radial profiles of the $m / n=0 / 0$ component of electron temperature (3a) and density (3b). The original equilibrium plasma rotation is in the electron drift direction with $\omega_{0}=-1.98$.

Fig. 4: Effect of parallel heat diffusion on radial profiles of the $m / n=0 / 0$ component of electron temperature (4a) and density (4b). The original equilibrium plasma rotation is in the ion drift direction with $\omega_{0}=2.97$.

Fig. 5: Effect of equilibrium plasma rotation direction and frequency on radial profiles of the $\mathrm{m} / \mathrm{n}=0 / 0$ component of electron temperature (5a) and density (5b) for $\psi_{\mathrm{a}}=8 \times 10^{-5}$ with $\omega_{0}=2.97,1.0,-1.98$ and 0 . Fig. 6: Effect of the parameter $d_{1}$ on radial profiles of the $m / n=0 / 0$ component of electron temperature (6a) and density (6b) for $\psi_{\mathrm{a}}=8 \times 10^{-5}$. The equilibrium plasma rotation is in the ion drift direction with $\omega_{0}=2.97$

Fig. 7: Effect of the perpendicular transport coefficients $\chi_{\perp}$ and $D_{\perp}$ on radial profiles of the $m / n=0 / 0$ component of electron temperature (7a) and density (7b) for $\psi_{\mathrm{a}}=8 \times 10^{-5}$. The equilibrium plasma rotation is in the ion drift direction with $\omega_{0}=2.97$.

Fig. 8: Radial profiles of the (normalized) $\mathrm{m} / \mathrm{n}=0 / 0$ component of poloidal plasma rotation velocity (8a), electron temperature (8b) and density (8c). The equilibrium plasma rotation is in the ion drift direction with $\omega_{0}=2.97$.

Fig. 9: Radial profiles of the (normalized) $\mathrm{m} / \mathrm{n}=0 / 0$ component of poloidal plasma rotation velocity 
(9a), electron temperature (9b) and density (9c). The equilibrium plasma rotation is in the electron drift direction with $\omega_{0}=-1.98$.

Fig. 10: Radial profiles of the (normalized) $\mathrm{m} / \mathrm{n}=0 / 0$ component of poloidal plasma rotation velocity (10a), electron temperature (10b) and density (10c). The original equilibrium plasma rotation speed is zero.

Fig. 11 Radial profiles of the $\mathrm{m} / \mathrm{n}=0 / 0$ component of electron density for original plasma rotation in the ion drift direction $\left(11 \mathrm{a}, \omega_{0}=2.92\right)$ and in the electron's direction $\left(11 \mathrm{~b}, \omega_{0}=-1.62\right)$. 


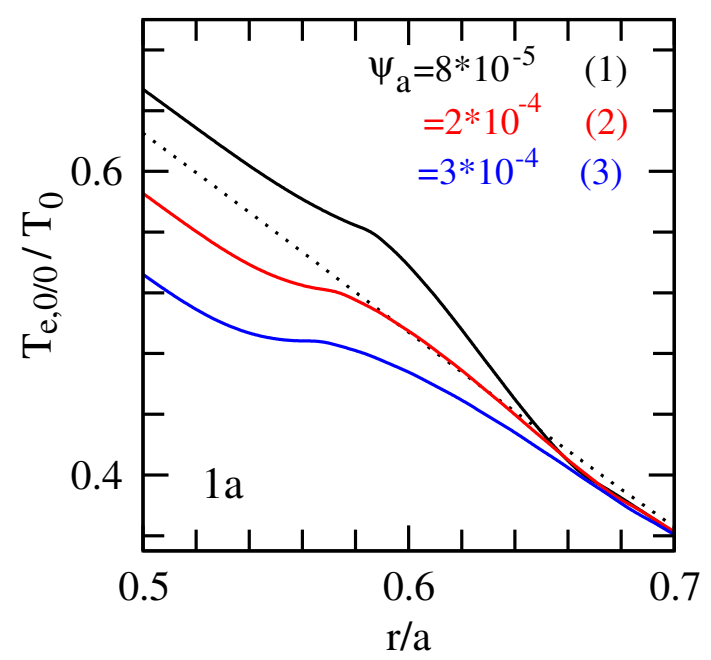




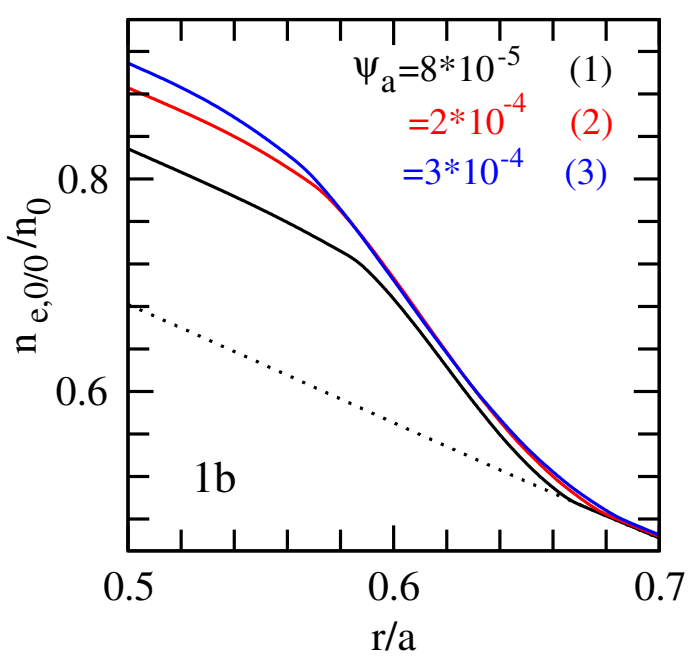




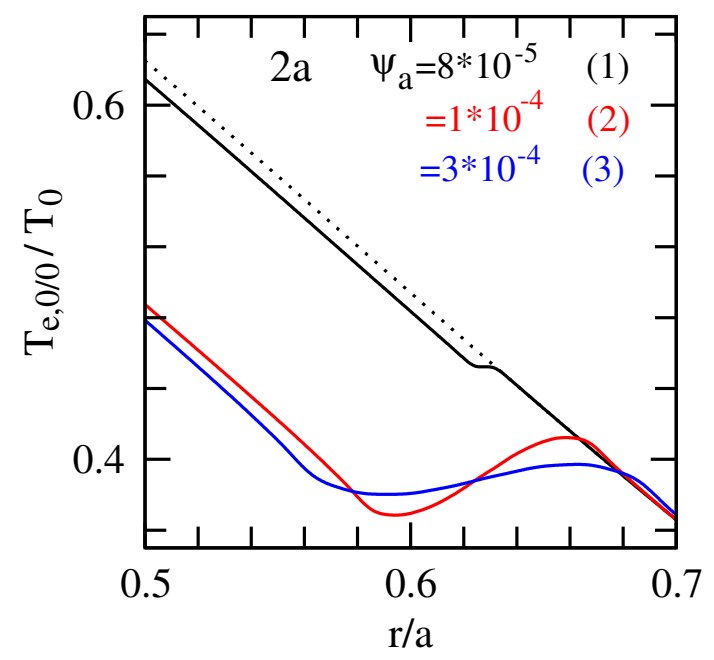




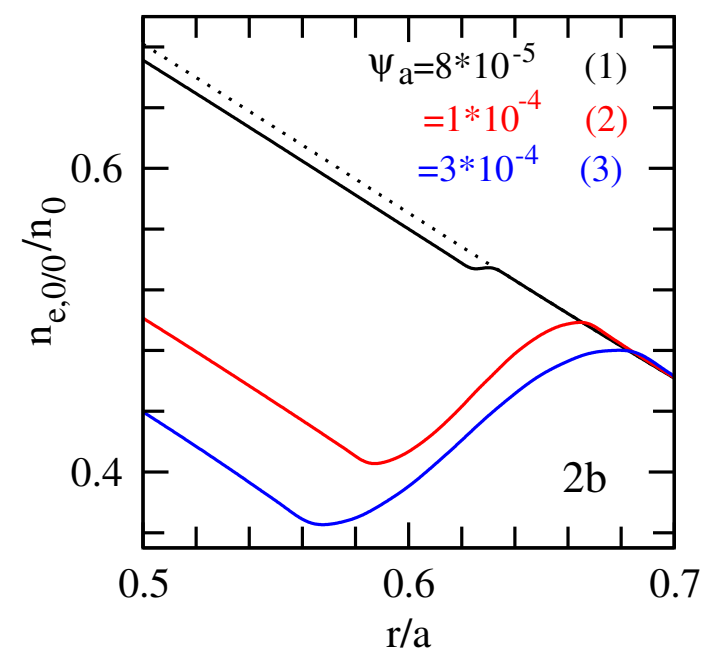




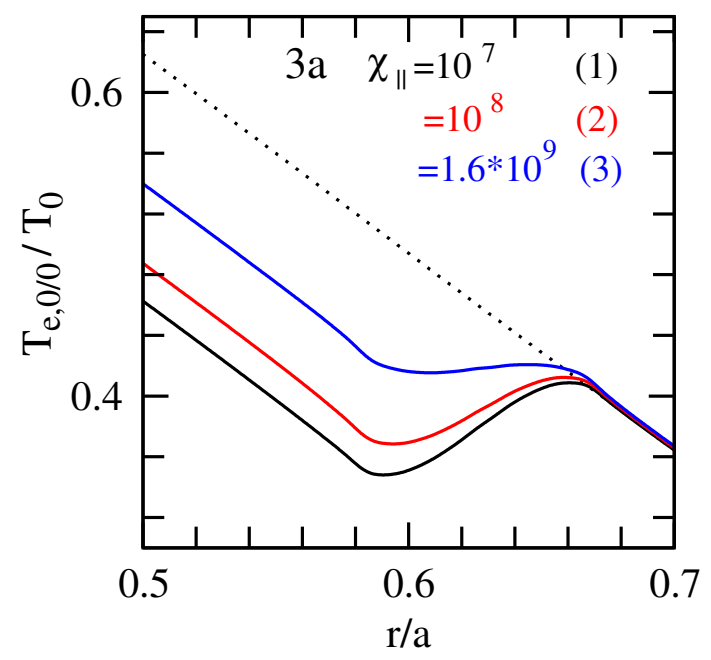




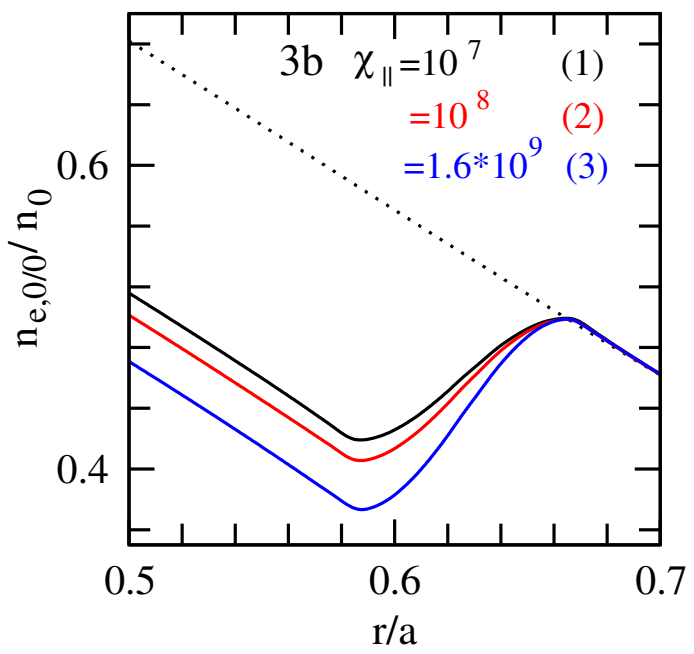




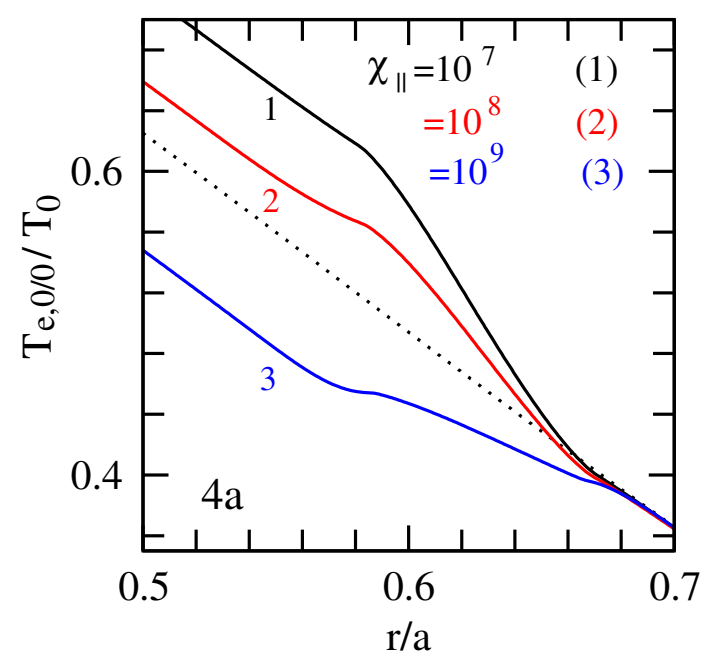




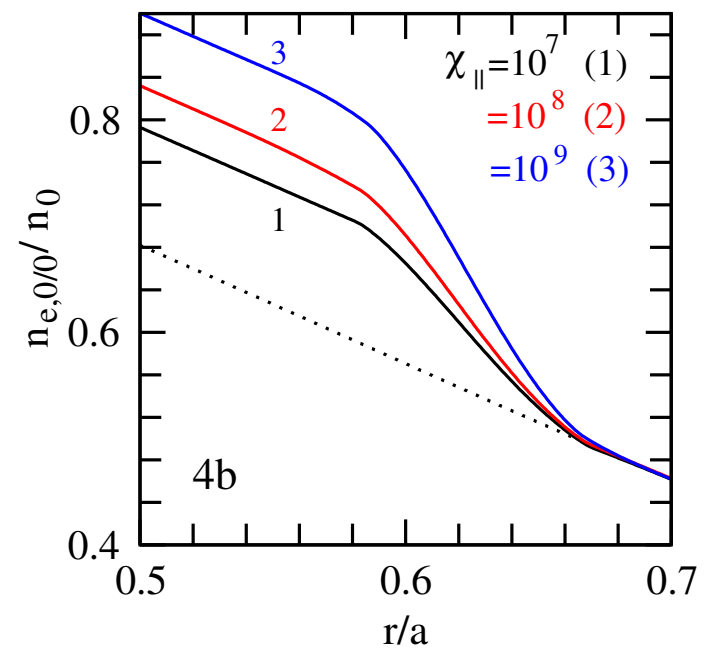




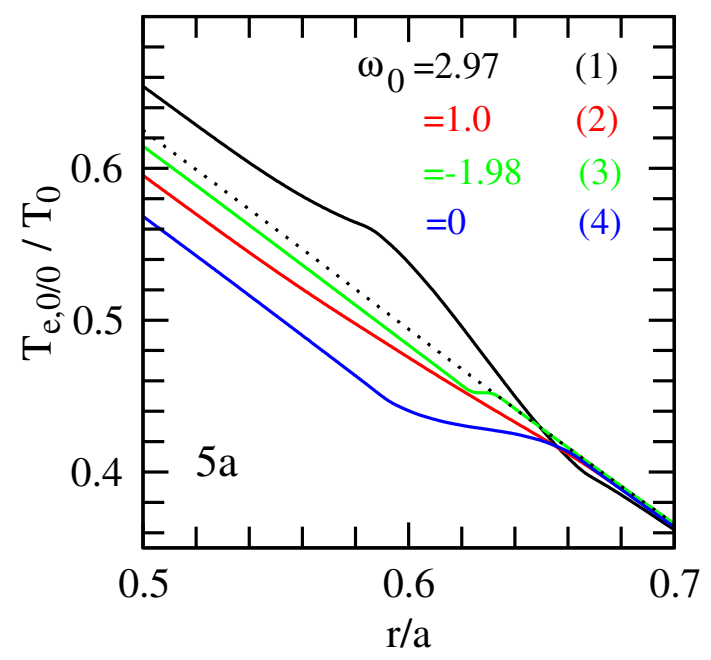




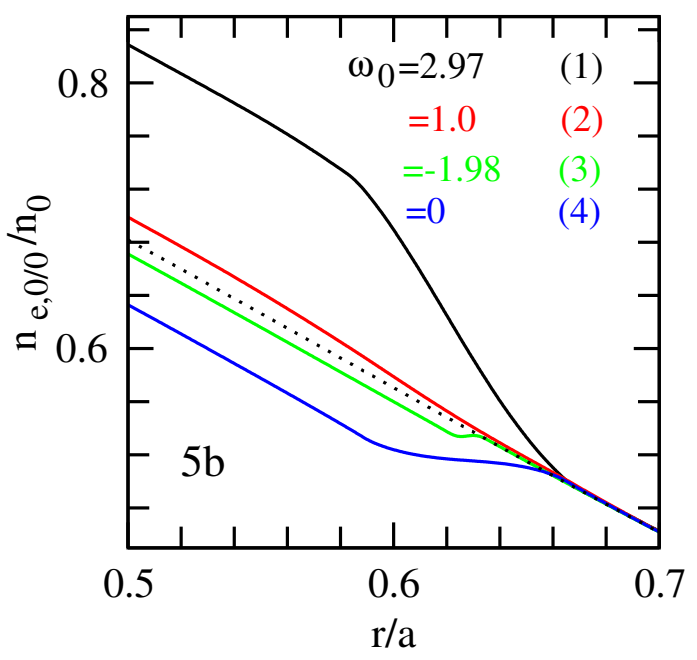




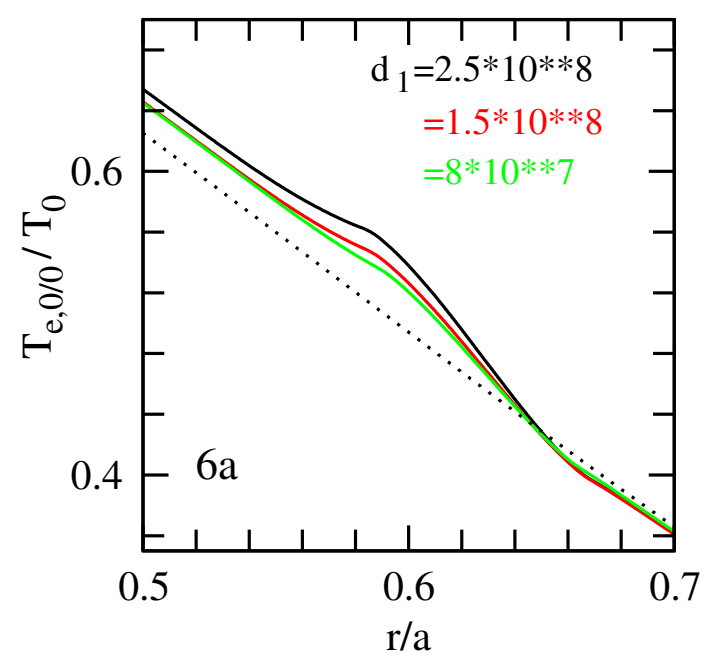




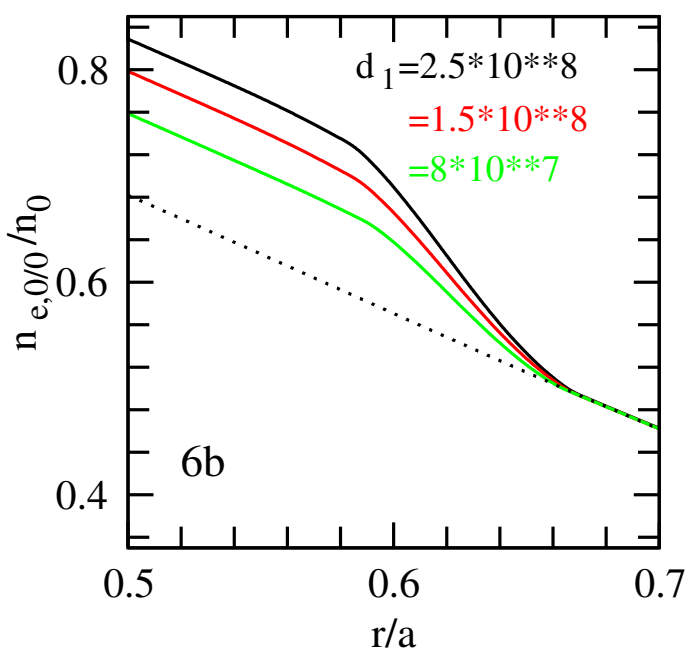




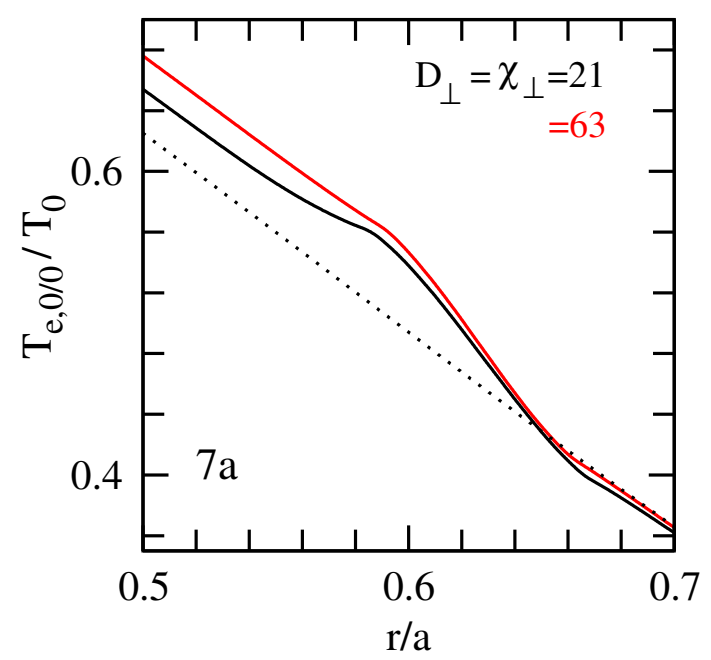




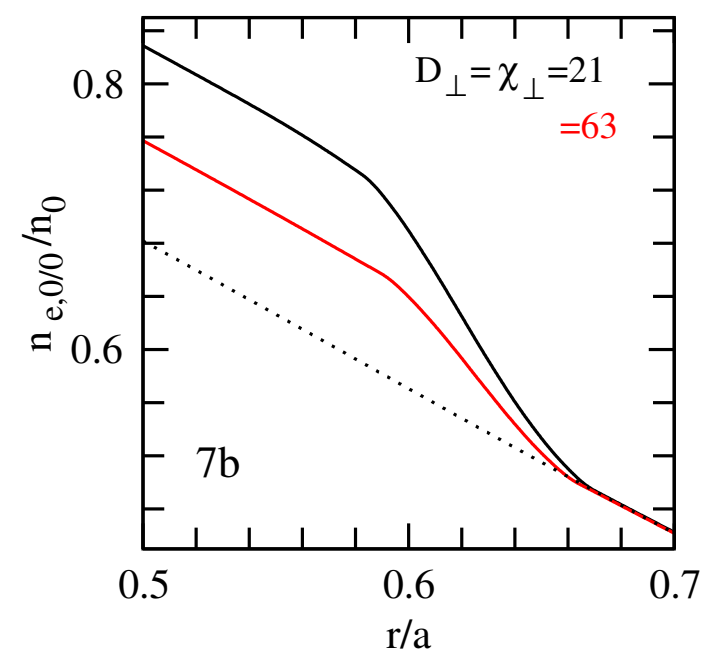




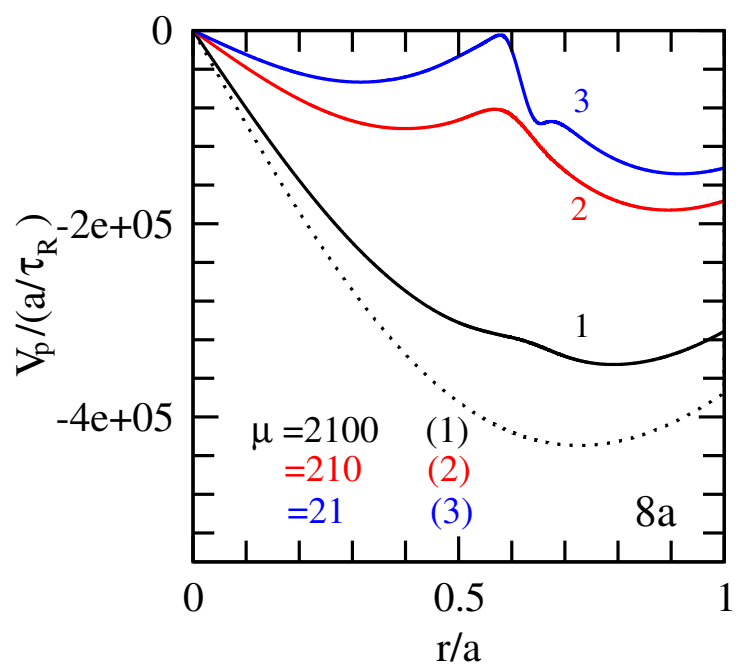




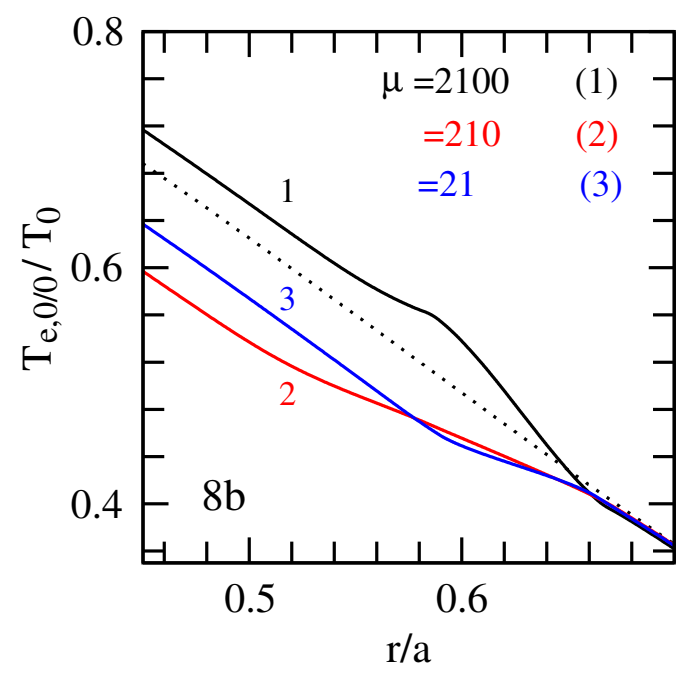




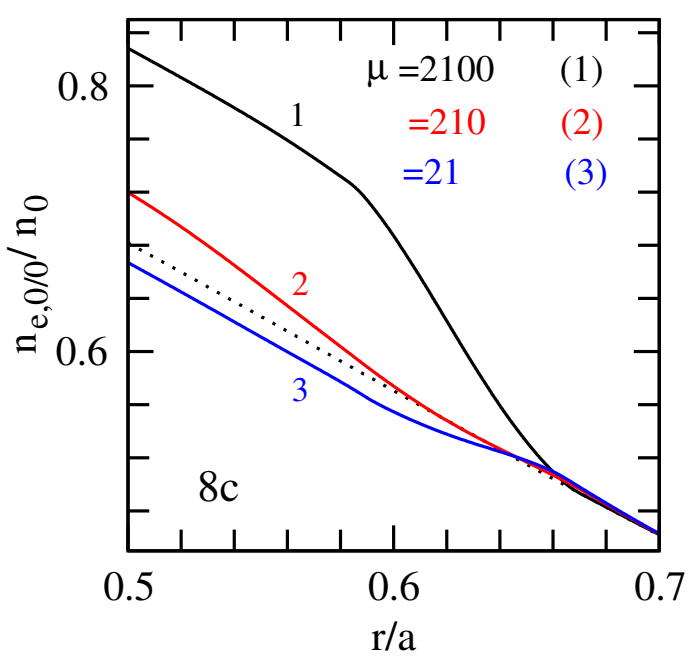




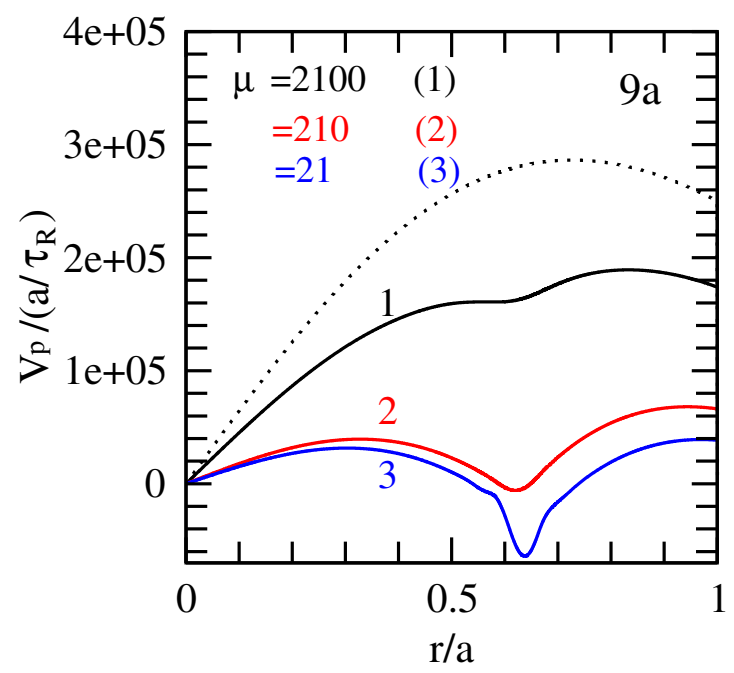




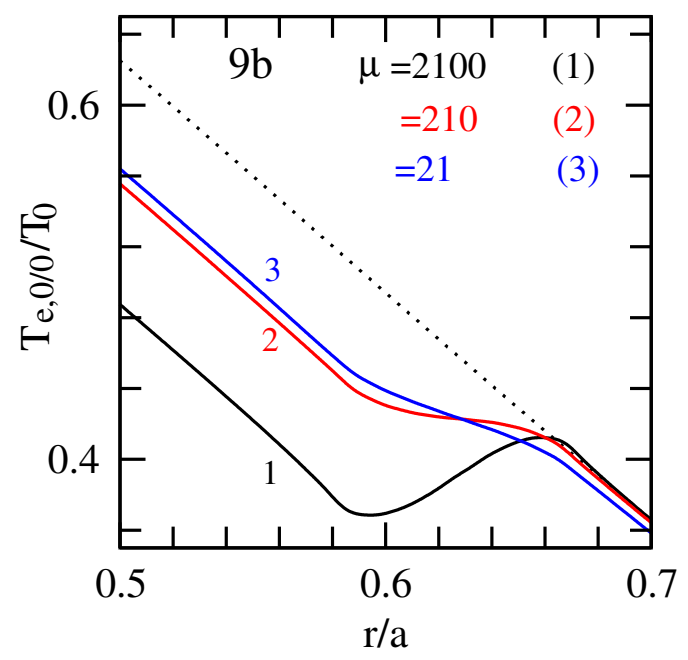




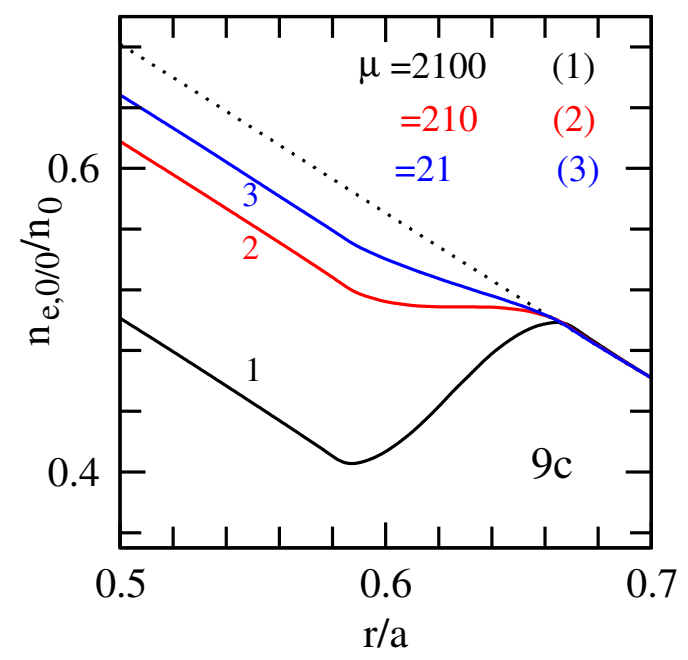




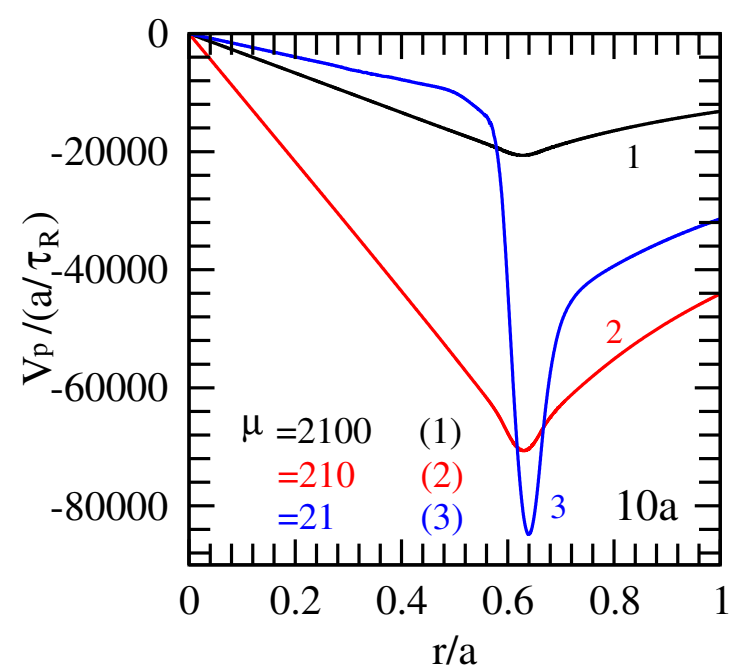




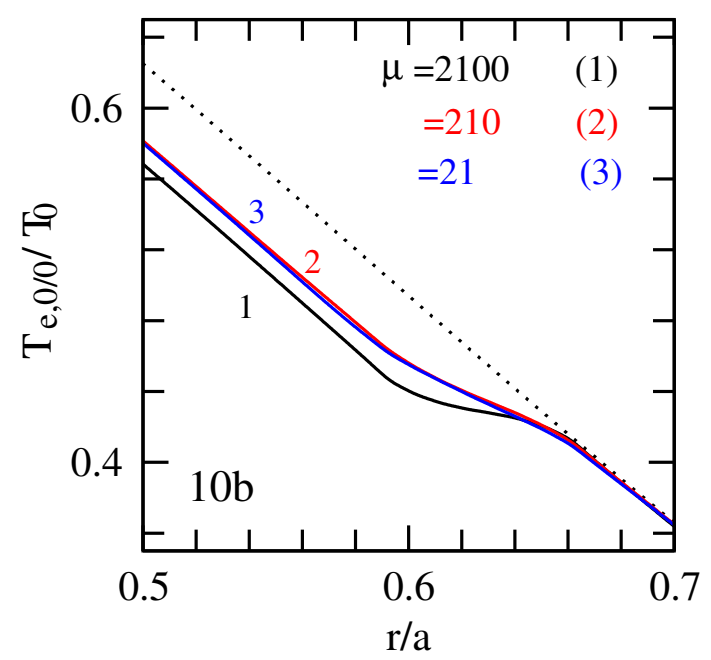




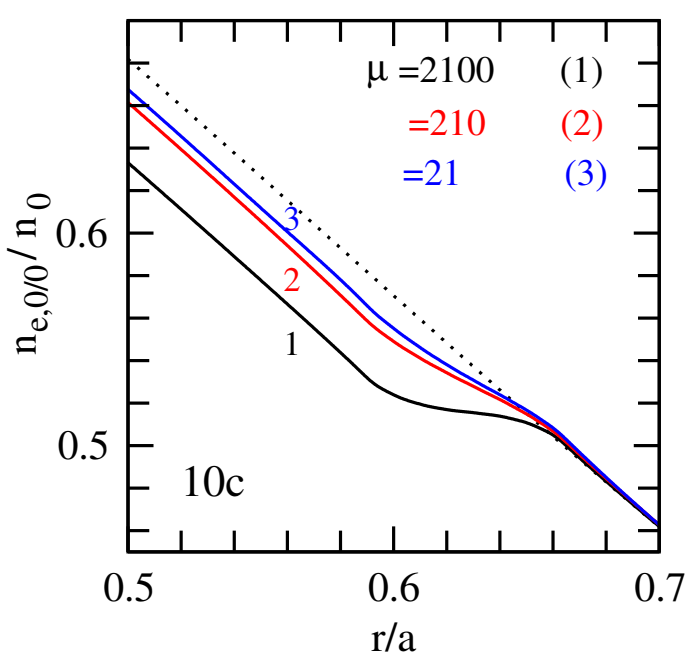




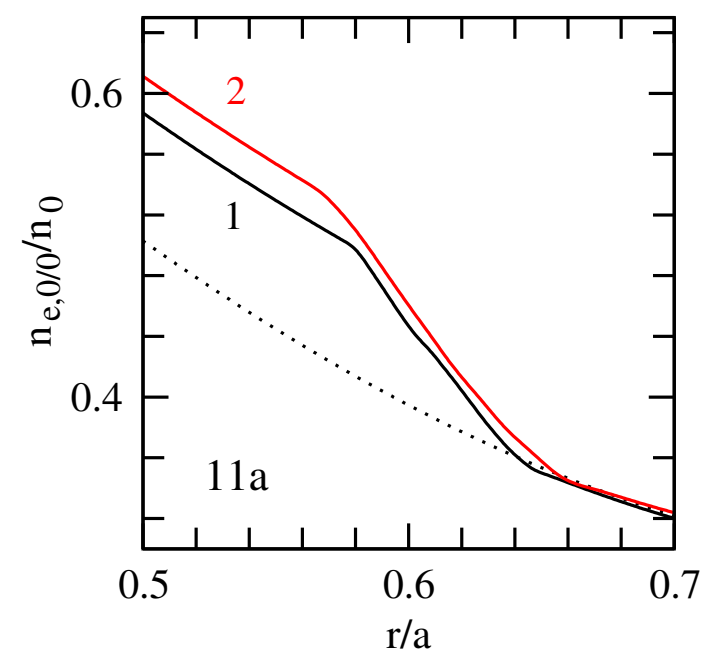




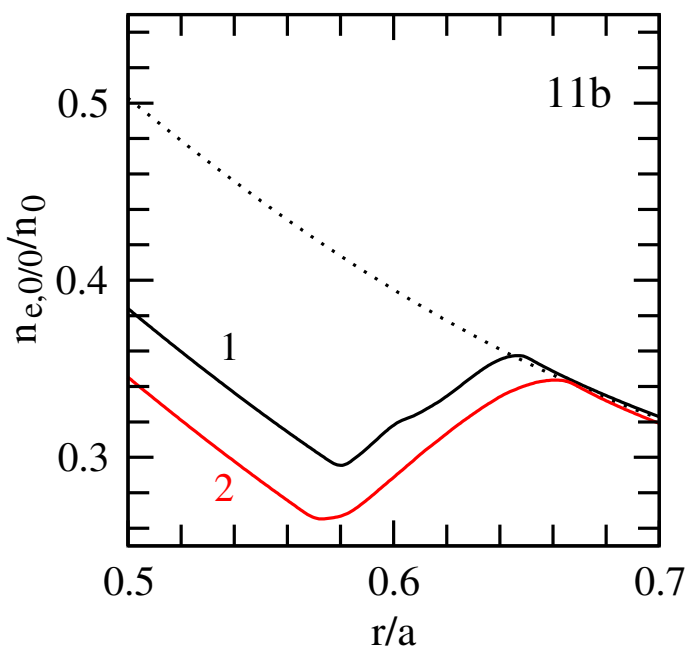

